\title{
4-D Imaging and Modeling of Eta Carinae's Inner Fossil Wind Structures
}

\author{
Thomas I. Madura ${ }^{1}$, Theodore Gull ${ }^{2}$, Mairan Teodoro ${ }^{2,3}$, \\ Nicola Clementel ${ }^{4}$, Michael Corcoran ${ }^{2,3}$, Augusto Damineli ${ }^{5}$, \\ Jose Groh $^{6}$, Kenji Hamaguchi ${ }^{2,7}$, D. John Hillier ${ }^{8}$, Anthony Moffat ${ }^{9}$, \\ Noel Richardson ${ }^{10}$, Gerd Weigelt ${ }^{11}$, Don Lindler ${ }^{2,12}$ \\ and Keith Feggans ${ }^{2,12}$ \\ ${ }^{1}$ San José State University, \\ One Washington Square, San José, CA 95192-0106, USA \\ email: thomas.madura@sjsu.edu \\ ${ }^{2}$ NASA Goddard Space Flight Center, Greenbelt, MD 20771, USA \\ ${ }^{3}$ USRA, 7178 Columbia Gateway Drive, Columbia, MD 20146, USA \\ ${ }^{4}$ South African Astronomical Observatory, P.O. Box 9, Observatory 7935, South Africa \\ ${ }^{5}$ IAG-USP, Rua do Matao 1226, Cidade Universitaria, Sao Paulo 05508-900, Brazil \\ ${ }^{6}$ Trinity College Dublin, University of Dublin, Dublin 2, Ireland \\ ${ }^{7}$ University of Maryland, Baltimore County, 1000 Hilltop Circle, Baltimore, MD 21250, USA \\ ${ }^{8}$ University of Pittsburgh, 3941 OHara Street, Pittsburgh, PA 15260, USA \\ ${ }^{9}$ Universite de Montreal, CP 6128 Succ. A., Centre-Ville, Montreal, Quebec H3C 3J7, Canada \\ ${ }^{10}$ University of Toledo, Toledo, OH 43606-3390, USA \\ ${ }^{11}$ Max-Planck-Institut fur Radioastronomie, Auf dem Hugel 69, D-53121 Bonn, Germany \\ ${ }^{12}$ Sigma Space Corporation, 4600 Forbes Blvd., Lanham, MD 20706, USA
}

\begin{abstract}
Eta Carinae is the most massive active binary within 10,000 light-years and is famous for the largest non-terminal stellar explosion ever recorded. Observations reveal that the supermassive $\left(\sim 120 \mathrm{M}_{\odot}\right)$ binary, consisting of an LBV and either a WR or extreme $\mathrm{O}$ star, undergoes dramatic changes every 5.54 years due to the stars' very eccentric orbits $(e \approx 0.9)$. Many of these changes are caused by a dynamic wind-wind collision region (WWCR) between the stars, plus expanding fossil WWCRs formed one, two, and three 5.54-year cycles ago. The fossil WWCRs can be spatially and spectrally resolved by the Hubble Space Telescope/Space Telescope Imaging Spectrograph (HST/STIS). Starting in June 2009, we used the HST/STIS to spatially map Eta Carinae's fossil WWCRs across one full orbit, following temporal changes in several forbidden emission lines (e.g. [Fe III] $4659 \AA$, [Fe II] $4815 \AA$ ), creating detailed data cubes at multiple epochs. Multiple wind structures were imaged, revealing details about the binary's orbital motion, photoionization properties, and recent $(\sim 5-15$ year $)$ mass-loss history. These observations allow us to test 3 -D hydrodynamical and radiative-transfer models of the interacting winds. Our observations and models strongly suggest that the wind and photoionization properties of Eta Carinae's binary have not changed substantially over the past several orbital cycles. They also provide a baseline for following future changes in Eta Carinae, essential for understanding the late-stage evolution of this nearby supernova progenitor. For more details, see Gull et al. (2016) and references therein.
\end{abstract}

Keywords. stars: individual (Eta Carinae), stars: mass loss, stars: winds, outflows

\section{Reference}

Gull, T. R., Madura, T. I., Teodoro, M., et al. 2016, MNRAS, 462, 3196 